\title{
Anti-proliferative action of IL-6R-targeted antibody tocilizumab for non-small cell lung cancer cells
}

\author{
NA-HYUN KIM, SEONG-KWAN KIM, DONG-SOON KIM, DAN ZHANG, \\ JIN-A PARK, HEE YI, JIN-SUK KIM and HO-CHUL SHIN \\ Department of Veterinary Pharmacology and Toxicology, College of Veterinary Medicine, \\ Konkuk University, Seoul 143-701, Republic of Korea
}

Received May 2, 2014; Accepted January 13, 2015

DOI: $10.3892 / 01.2015 .3019$

\begin{abstract}
In the present study we analyzed the anti-proliferative effect of tocilizumab, a humanized recombinant monoclonal interleukin 6 receptor (IL-6R) antibody, against non-small cell lung cancer (NSCLC) cells, including A549, H460, H358 and H1299 cells. The cell cycle distribution of NSCLCs was analyzed using fluorescence-activated cell sorting and gene expression using quantitative polymerase chain reaction. Cell lysates treated with tocilizumab were immunoblotted with antibodies against signal transducer and activator of transcription 3 (STAT3), phospho-STAT3, extracellular-signal-regulated kinases (ERK), phospho-ERK, nuclear factor $\kappa \mathrm{B}(\mathrm{NF} \kappa \mathrm{B})$ and phospho-NFKB. Significant growth inhibition of NSCLC cells was observed following treatment with tocilizumab. Proliferation was significantly decreased by approximately $10-40 \%$ in A549, H460, H1299 and H358 cells, with an inhibition rate that was comparable with that of the typical anticancer drugs methotrexate and 5-fluorouracil. NSCLC cell populations were accumulated in the sub-G1 phase by treatment with tocilizumab. Western blot analyses revealed a possible activation of the NFkB pathway by tocilizumab. Overall, these data indicate that tocilizumab has anticancer potency via apoptosis induction as an agonistic IL-6R regulator. Therefore, we suggest that this anti-IL-6R antibody may be utilized as a new targeting molecule for NSCLC therapies.
\end{abstract}

\section{Introduction}

Non-small cell lung cancer (NSCLC) is the most common type of lung cancer, accounting for more than $80 \%$ of cases. As a class, NSCLC is relatively insensitive to chemotherapy compared with small cell carcinoma. A number of drugs are

Correspondence to: Professor Ho-Chul Shin, Department of Veterinary Pharmacology and Toxicology, College of Veterinary Medicine, Konkuk University, 120 Neungdong-ro, Gwangjin-gu, Seoul 143-701, Republic of Korea

E-mail: hshin@konkuk.ac.kr

Key words: tocilizumab, interleukin 6 signaling, non-small cell lung cancer, $\mathrm{NF \kappa B}$, anti-proliferation useful for NSCLC as systemic therapy, including platinum agents (cisplatin and carboplatin), taxanes (paclitaxel and docetaxel), vinorelbine, vinblastine, etoposide, pemetrexed and gemcitabine. However, despite advances in chemotherapeutic drugs, the prognosis of lung cancer remains poor, with an overall 5-year survival rate of less than $14 \%$ in the USA and even lower (5-10\%) in Europe and other countries (1).

A number of investigators have attempted to develop specific targeting molecules, including antibodies and antagonists, for the treatment of NSCLC. Bevacizumab is a recombinant monoclonal antibody that blocks vascular endothelial growth factor. Erlotinib is a small molecule inhibitor of epidermal growth factor receptor (EGFR), and crizotinib is a small molecule inhibitor that targets anaplastic lymphoma kinase and hepatocyte growth factor receptor c-MET. Cetuximab is a monoclonal antibody that targets EGFR.

The pathway of interleukin 6/interleukin 6 receptor (IL-6/IL-6R) signaling regulates diverse biological activities, including cell growth and differentiation, in immune and hematopoietic systems (2). However, IL-6/IL-6R signaling is also known to promote tumor growth and survival in several organ systems (3). The plasma IL-6 levels of cancer patients were observed to correlate with the clinical and pathological variety and survival in prostate cancer (4), ovarian cancer (5), breast cancer (6) and colorectal cancer (7). Tocilizumab is a humanized recombinant $\mathrm{IgG1k}$ monoclonal antibody against IL-6R (8). It is approved by the FDA and used in clinics to treat rheumatoid arthritis and juvenile idiopathic arthritis patients (9). It has also been demonstrated that tocilizumab has an anti-proliferative effect on glioma cells via inhibition of the JAK-STAT3 pathway (10). Furthermore, there is evidence that IL-6/IL-6 signaling affects NSCLC tumorigenesis (11).

In a previous study (12), we demonstrated the possibility of using IL-6R as a selective anticancer drug target using an H460 lung cancer stem cell model. Here, we investigate the effect of IL-6R blockage on the proliferation of NSCLC cells using the IL-6R-targeted antibody tocilizumab.

\section{Materials and methods}

Cell culture. NSCLC cell lines H460, A549, H1299 and H358 were obtained from the Korean Cell Line Bank (KCLB, 
Seoul, Korea). The cells were grown in RPMI-1640 medium (Gibco Life Technologies, Carlsbad, CA, USA) with 10\% heat-inactivated fetal bovine serum (Gibco) and $1 \%$ penicillin/ streptomycin (Gibco) at $37^{\circ} \mathrm{C}$ in a humidified incubator $(5 \%$ $\mathrm{CO}_{2}$ ), as recommended by KLCB.

Cell proliferation assay. The anticancer drugs methotrexate (MTX) and 5-fluorouracil (5-FU) were purchased from Sigma-Aldrich (St. Louis, MO, USA), and tocilizumab was kindly donated by Dr Misahiko Mihara at Chugai Pharmaceutical Co. Ltd. (Shizuoka, Japan). The anti-proliferation activity of tocilizumab, MTX and 5-FU in NSCLC cells in vitro was measured using the EZ-Cytox kit (Daeillab, Seoul, Korea). Ten microliters of tocilizumab, MTX or 5-FU were added to 96-well plates containing $10^{4}$ cells per well in $100 \mu \mathrm{l}$ medium. The final concentrations of tocilizumab were 10, 100 and $1000 \mathrm{ng} / \mathrm{ml}$. The final concentrations of MTX and 5-FU were 50 and $25 \mu \mathrm{g} / \mathrm{ml}$, respectively. Following a 24-h incubation, WST-1 solution (Daeillab) was added, and the optical density was analyzed using the ELISA plate reader Magellan ${ }^{\mathrm{TM}}$ (Tecan, Männedorf, Switzerland) at reference wavelengths of 450 and $620 \mathrm{~nm}$.

Cell cycle analysis. The NSCLC cells were seeded at $2.0 \times 10^{5}$ cells/well in 6 -well plates. The cells were allowed to recover for $24 \mathrm{~h}$ and then treated with tocilizumab. To analyze the cell cycle distribution, the cells were collected after a 24-h incubation and washed with phosphate-buffered saline (PBS). The cells were fixed in $70 \%$ ethanol and stored overnight at $4^{\circ} \mathrm{C}$. For the analysis, the cells were transferred to PBS and incubated with ribonuclease $\mathrm{A}(50 \mu \mathrm{g} / \mathrm{ml})$ at room temperature for $5 \mathrm{~min}$. The cells were then stained with $10 \mu \mathrm{g} / \mathrm{ml}$ propidium iodide (PI) and incubated at $37^{\circ} \mathrm{C}$ for $10 \mathrm{~min}$. Finally, the cells were analyzed using fluorescence-activated cell sorting.

RNA extraction and quantitative polymerase chain reaction (qPCR). qPCR was performed to identify the gene expression level of IL-6R in the NSCLC cells based on the expression of a housekeeping gene, glyceraldehyde-3-phosphate dehydrogenase (GAPDH), as an internal control. RNA was quantified by its absorption at $260 \mathrm{~nm}$ and stored at $-80^{\circ} \mathrm{C}$ before use. Briefly, first-strand cDNA was synthesized from $2 \mu \mathrm{g}$ total RNA with Superscript III transcriptase (Invitrogen Life Technologies, Carlsbad, CA, USA). PCR amplification was performed with specific primer pairs designed from published human gene sequences (13). qPCR was performed using SYBR-Green (Takara Bio Inc., Shiga, Japan) and a Bio-Rad machine (Bio-Rad Laboratories Inc., Hercules, CA, USA). DNA was amplified using 60 cycles of denaturation for $5 \mathrm{sec}$ at $95^{\circ} \mathrm{C}$ and annealing for $40 \mathrm{sec}$ at $60^{\circ} \mathrm{C}$.

Protein extraction and western blot analysis. Whole-cell lysates were extracted using the Pro-Prep protein extraction solution plus protease inhibitor cocktail (Intron Biotechnology, Seongnam, Korea) according to the method described in the manufacturer's guidelines. Cell lysates were separated using $10 \%$ sodium dodecyl sulfate-polyacrylamide gel electrophoresis (SDS-PAGE), transferred to a nitrocellulose membrane (Bio-Rad), and immunoblotted with antibodies against the following: signal transducer and activator of transcription 3
(STAT3), phospho-STAT3, extracellular-signal-regulated kinases (ERK), phospho-ERK, nuclear factor $\kappa \mathrm{B}(\mathrm{NF \kappa B})$ and phospho-NFкB (Cell Signaling Technology, Inc., Beverly, MA, USA). After incubating with the secondary antibody, the membranes were developed using enhanced chemiluminescence. ImageJ software (NIH, USA) was used to analyze the results.

Statistical analysis. The results are expressed as the means \pm standard deviation. Analysis of variance was used to compare differences among the groups. $\mathrm{P}<0.05$ was considered to indicate a statistically significant difference. Statistical analyses were performed with Statistical Analysis Systems software (SPSS version 20; IBM SPSS, Armonk, NY, USA).

\section{Results}

Cell proliferation. H460, A549, H1299 and H358 cells were treated in triplicate with tocilizumab at concentrations of 10,100 and $1000 \mathrm{ng} / \mathrm{ml}$. The inhibition of cell growth was examined by a commercial kit and an ELISA reading system after $24 \mathrm{~h}$ of treatment and was calculated as the percentage of viable cells relative to untreated cell cultures. As shown in Fig. 1A, tocilizumab demonstrated substantial growth inhibition in the NSCLC cells. Following exposure to tocilizumab at a $100 \mathrm{ng} / \mathrm{ml}$ concentration, cell growth was significantly decreased by $27.75 \pm 5.81,34.23 \pm 9.49,22.14 \pm 4.87$ and $10.81 \pm 1.94 \%$ in the H460, A549, H1299 and H358 cells, respectively. In addition, the anti-proliferative effect of tocilizumab $(100 \mathrm{ng} / \mathrm{ml})$ was compared with that of the conventionally used anticancer drugs MTX and 5-FU in the NSCLC cells. The concentrations of MTX (50 mg/ml) and 5-FU (25 mg/ml) were based on those used in our previous study (12). MTX is a novel drug that acts as an inhibitor of folate metabolism, and 5-FU is an irreversible inhibitor of thymidylate synthase. These drugs have been used in the treatment of NSCLC patients for some time. As shown in Fig. 1B, the cell growth inhibition rate of tocilizumab in the NSCLC cells was similar or only slightly lower than that of MTX and 5-FU.

Cell cycle distribution. A flow cytometric cell cycle analysis was performed to determine whether the results of the cell assay reflected cytostatic or cytotoxic effects due to cell cycle arrest or apoptosis. NSCLC cells were treated with $100 \mathrm{ng} / \mathrm{ml}$ tocilizumab. After $24 \mathrm{~h}$ of drug treatment, the cells were fixed and suspended in PI and measured in comparison with untreated cells. Morphological changes in the apoptotic cells, including shrinkage, rounding and membrane blebbing, were also observed following the tocilizumab treatment. As shown in Fig. 2, a significant accumulation of the cell population in the sub-G1 phase was observed in the H460 and A549 cells following drug treatment. Similar effects on the cell cycle were observed with tocilizumab concentrations of 10 and $1000 \mathrm{ng} / \mathrm{ml}$. Following tocilizumab treatment, $8.73 \%$ of the $\mathrm{H} 460$ cell population was arrested in the sub-G1 phase in contrast to $0.21 \%$ of the untreated control cells (Fig. 2A). In addition, $25.01 \%$ of the A549 cell population was in the sub-G1 phase following drug treatment, compared with only $1.74 \%$ of the untreated control cells (Fig. 2B). Although there was no significant difference in the H358 and H1299 cells between the 

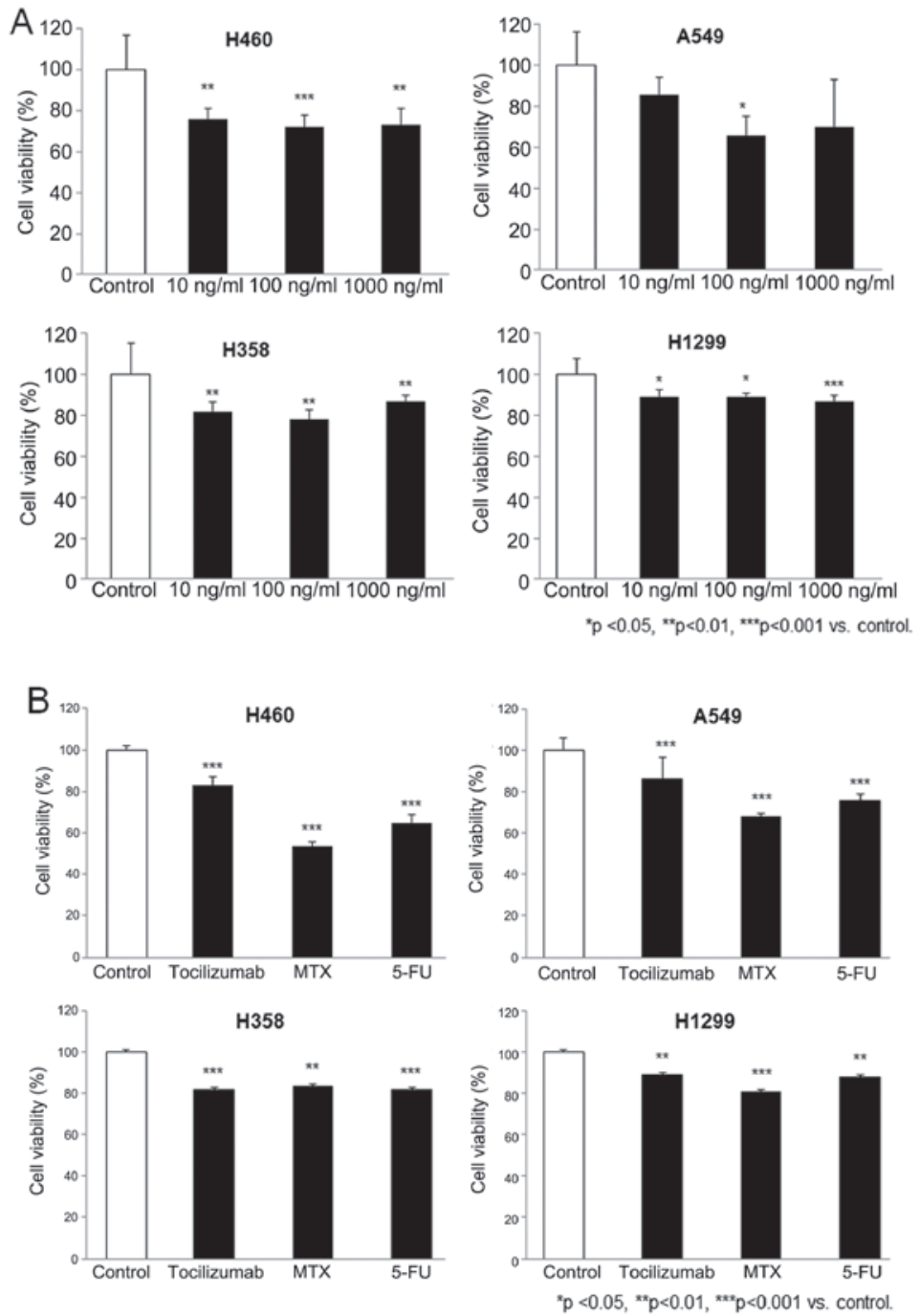

Figure 1. Growth inhibitory activity of tocilizumab against non-small cell lung cancer cell lines: H460, A549, H1299 and H358 cells. The cells were treated with the indicated concentrations of tocilizumab for $24 \mathrm{~h}$ (A). The percent cell viability was determined by WST-1 assay. The anti-proliferative effect of tocilizumab was also compared with that of typical anticancer drugs methotrexate (MTX) and 5-fluorouracil (5-FU). (B) Data are expressed as the means \pm SEM (n=4). ${ }^{*} \mathrm{P}<0.05,{ }^{* *} \mathrm{P}<0.01,{ }^{* * *} \mathrm{P}<0.001$ vs. control.

A

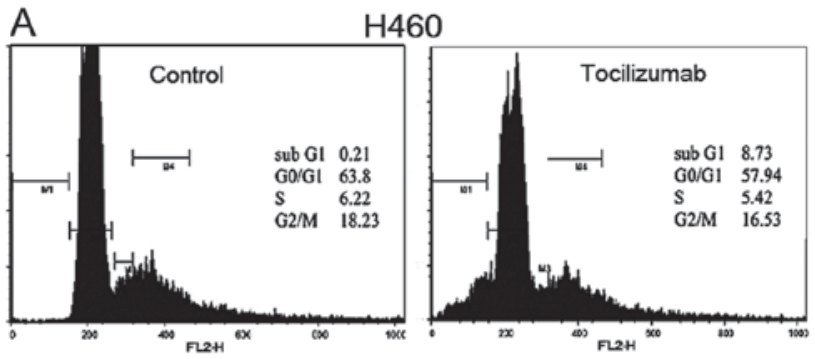

C

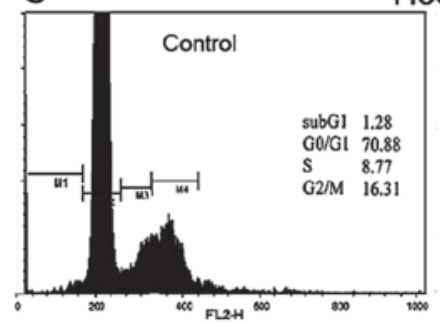

$\mathrm{H} 358$

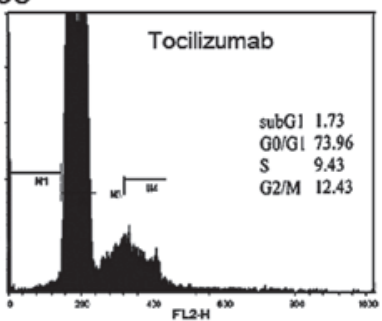

B

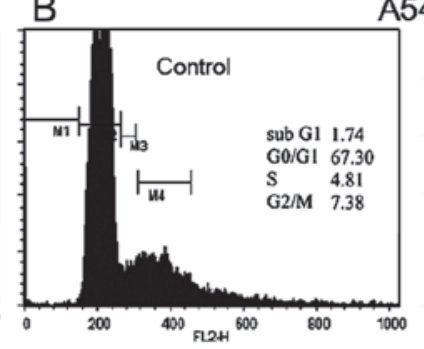

A549
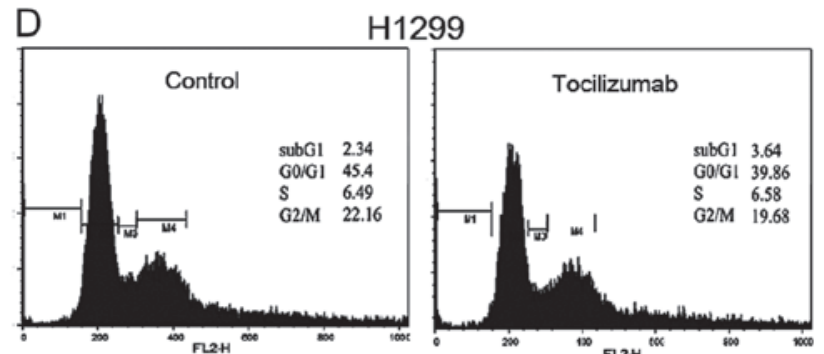

Figure 2. Effect of tocilizumab on cell cycle distribution in non-small cell lung cancer (NSCLC) cells. NSCLC cells (A) H460, (B) A549, (D) H1299 and (C) H358 were treated with $100 \mathrm{ng} / \mathrm{ml}$ tocilizumab for $24 \mathrm{~h}$. The cell cycle distribution was analyzed by flow cytometry. The percentage of cells in the G0/G1, $\mathrm{S}$ and G2/M phases of the cell cycle are expressed as the means \pm SEM ( $n=3)$. M1, sub-G1; M2, G0/G1; M3, S; M4, G2/M. 


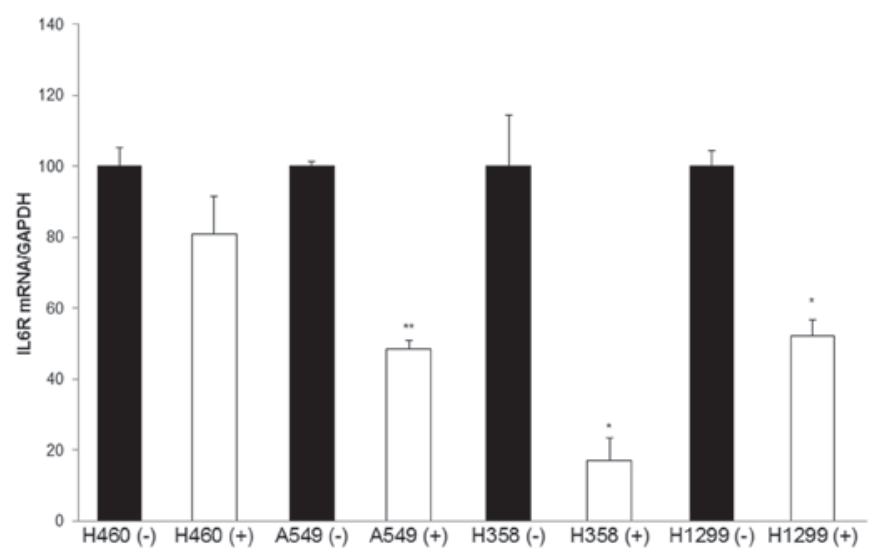

Figure 3. Regulation of interleukin-6 receptor (IL-6R) mRNA levels in non-small cell lung cancer cells H460, A549, H358 and H1299 following tocilizumab treatment at $100 \mathrm{ng} / \mathrm{ml}$. The mRNA levels were analyzed by quantitative polymerase chain reaction. GAPDH, glyceraldehyde-3-phosphate dehydrogenase; (+) tocilizumab treatment; (-) no tocilizumab treatment. ${ }^{~} \mathrm{P}<0.05$ vs. the non-treated group, ${ }^{* *} \mathrm{P}<0.01$ vs. the non-treated group.

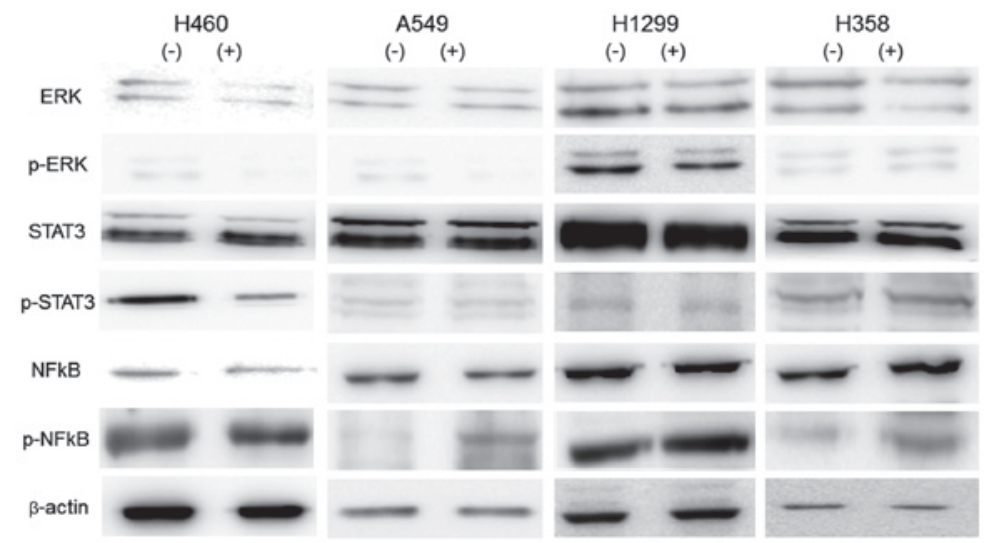

Figure 4. Tocilizumab activates the nuclear factor $\kappa \mathrm{B}(\mathrm{NF} \kappa \mathrm{B})$ pathway. Cell lysates were immunoblotted with antibodies against signal transducer and activator of transcription 3 (STAT3), phospho-STAT3 (p-STAT3), extracellular-signal-regulated kinases (ERK), phospho-ERK (p-ERK), nuclear factor $\kappa B$ (NFkB) and phospho-NFkB (p-NFkB). (+) Tocilizumab treatment; (-) no tocilizumab treatment.

treated and untreated groups, minor increases were observed following tocilizumab treatment: $1.73 \%$ of $\mathrm{H} 358$ cells were in the sub-G1 phase compared with $1.28 \%$ of the untreated control cells (Fig. 2C), and 3.64\% of H1299 cells were in the sub-G1 phase compared with $2.34 \%$ of the untreated control cells (Fig. 2D).

Gene expression and immunoblotting. The mRNA expression of IL-6R was analyzed in H460, A549, H1299 and H358 cells using qPCR. The transcript levels were normalized to the expression of GAPDH. The data in Fig. 3 reveal marked decreases in IL-6R expression with tocilizumab at a concentration of $100 \mathrm{ng} / \mathrm{ml}$. Tocilizumab significantly reduced the mRNA levels of IL-6R by $50 \%$ in A549, $77 \%$ in $\mathrm{H} 358,48 \%$ in $\mathrm{H} 1299$, and $20 \%$ in $\mathrm{H} 460$ cells. The principal transcriptional factors in the IL-6R/IL-6 signaling pathway regulating cell differentiation and growth are ERK1/2, STAT3 and $\mathrm{NF}_{\kappa} \mathrm{B}$. Thus, we estimated the influence of tocilizumab on the levels of activation of these factors in NSCLC cells. The cells were treated with $100 \mathrm{ng} / \mathrm{ml}$ tocilizumab, and protein lysates were obtained after $24 \mathrm{~h}$ of drug exposure. The levels of the transcription factors and their phosphorylated forms in whole-cell lysates were analyzed by western blotting using commercially available antibodies. As shown in Fig. 4, tocilizumab did not alter the levels of the proteins ERK1/2, STAT3 and NFкB and phosphorylated ERK1/2 and STAT3. However, phosphorylated NFKB was considerably increased by tocilizumab treatment in the NSCLC cells.

\section{Discussion}

IL-6 plays a significant role in the neoplastic process through its action on cancer cell adhesion, motility, proliferation, tumor-specific antigen expression and thrombopoiesis (14). IL-6, with its known functions in immune response, acute phase reaction and hematopoiesis, was considered a therapeutic target approximately two decades ago. The aim of this study was to assess whether an anti-IL-6R antibody could be utilized as a new targeting molecule for NSCLC therapy. Another anti-IL-6 antibody, siltuximab, has been demonstrated to have potential benefits in the treatment of various human cancers, including multiple myeloma, breast cancer and prostate cancer, either as a single agent or in combination with other chemotherapy drugs (14-17). It has also been indicated that anti-IL-6R antibodies may be a potential agent for the suppression of colon cancer progression $(18,19)$. 
These earlier investigations have revealed IL-6R as a potent target for antibody treatment in anticancer therapy. To our knowledge, there has been no study describing the utilization of an anti-IL-6/IL-6R antibody in NSCLC cancer therapy.

Tocilizumab is a fully humanized monoclonal antibody against IL-6R that was approved for the treatment of patients with rheumatoid arthritis (20). In Japan, tocilizumab has also been approved for the treatment of polyarticular-course juvenile idiopathic arthritis, systemic-onset juvenile idiopathic arthritis and Castleman's disease (21). However, this drug was initially investigated in the field of oncology. Mouse monoclonal antibodies against human IL-6 were noted to be effective in a patient with plasma cell leukemia (22). The mouse monoclonal antibody bound to the human IL-6R, inhibiting IL-6 function, and demonstrated strong antitumor cell activity against multiple myeloma cells (23). In our study, tocilizumab exhibited a significant growth inhibition in NSCLC cells (H460, A549, H1299 and H358), with proliferation significantly decreased by approximately $40 \%$ in A549 cells. The growth inhibition rates of tocilizumab in NSCLC cells were comparable with those of MTX and 5-FU, classically used anticancer drugs. This finding indicates that tocilizumab has a potent antitumor activity against NSCLC. Kudo et al (10) described the antitumor effect of tocilizumab in U87MG glioma cells and the critical role of the IL-6 signaling pathway in glioma cell proliferation. We further examined whether the result of our cell assay was a reflection of cytostatic or cytotoxic effects due to cell cycle arrest. Compared with the untreated control, tocilizumab treatment resulted in an approximate 40-fold increase in sub-G1 phase arrest in H460 cells and an approximately 14-fold increase in A549 cells. H358 and H1299 cells also exhibited approximately 1.3- and 1.5-fold increases, respectively, in the cell population in the sub-G1 phase. The statistical accumulation of the cell population in the sub-G1 phase demonstrates that significant apoptosis occurred in NSCLC cells following tocilizumab treatment.

The mRNA expression of IL-6R was analyzed in NSCLC cells. Tocilizumab significantly reduced the mRNA levels of IL-6R by $20-80 \%$. Although we have no direct data for the downregulation of IL-6R at the protein level, the above results may indicate that tocilizumab has a regulatory function. It is known that ERK1/2, STAT3 and NFkB are involved in the signaling pathway of IL-6R/IL-6 as vital transcriptional factors in tumor proliferation (24). In our study, tocilizumab did not alter the levels of the ERK1/2, STAT3, NFKB and phosphorylated ERK1/2 and STAT3 proteins, but this antibody did considerably increase the expression of phosphorylated NFкB in NSCLC cells. This result indicates that the phosphorylation of $\mathrm{NF} \kappa \mathrm{B}$ may be an significant factor in the anti-proliferative activity of tocilizumab. NFאB is a key transcriptional regulator of genes involved in inflammatory responses as well as genes regulating cell proliferation and metastasis in carcinogenesis $(25)$. $\mathrm{NF} \kappa \mathrm{B}$ is considered to function either as an inhibitor or an activator of apoptotic cell death. The nuclear accumulation and transcriptional activity of $\mathrm{NF \kappa B}$ are increased in T-cell lymphoma cells and are responsible for their increased proliferation (26). However, evidence has also revealed a pro-apoptotic role for $\mathrm{NF} \kappa \mathrm{B}$. Martin (27) emphasized the balance of NFKB activation with regard to pro-apoptotic and anti-apoptotic effects at the level of target gene activation. Although a number of phosphorylation sites on $\mathrm{NF \kappa B}$ proteins have been characterized, it remains unclear how phosphorylation regulates the activities of related proteins and controls target gene expression (28).

Our study revealed the anti-proliferation potency of tocilizumab on NSCLC cells via apoptosis induction and IL-6R signaling alteration. Therefore, we suggest that the IL-6R antibody may be utilized as a new targeting molecule in NSCLC cancer therapies.

\section{Acknowledgements}

This paper was supported by Konkuk University in 2013.

\section{References}

1. Lam WK and Watkins DN: Lung cancer: future directions. Respirology 12: 471-477, 2007.

2. Kishimoto T, Akira S, Narazaki M and Taga T: Interleukin-6 family of cytokines and gp130. Blood 86: 1243-1254, 1995.

3. Hodge DR, Hurt EM and Farrar WL: The role of IL-6 and STAT3 in inflammation and cancer. Eur J Cancer 41: 2502-2512, 2005.

4. Michalaki V, Syrigos K, Charles P and Waxman J: Serum levels of IL-6 and TNF-alpha correlate with clinicopathological features and patient survival in patients with prostate cancer. Br J Cancer 90: 2312-2316, 2004.

5. Plante M, Rubin SC, Wong GY, Federici MG, Finstad CL and Gastl GA: Interleukin-6 level in serum and ascites as a prognostic factor in patients with epithelial ovarian cancer. Cancer 73: 1882-1888, 1994

6. Zhang GJ and Adachi I: Serum interleukin-6 levels correlate to tumor progression and prognosis in metastatic breast carcinoma. Anticancer Res 19: 1427-1432, 1999.

7. Chung YC and Chang YF: Serum interleukin-6 levels reflect the disease status of colorectal cancer. J Surg Oncol 83: 222-226, 2003.

8. Ohsugi Y and Kishimoto T: The recombinant humanized anti-IL-6 receptor antibody tocilizumab, an innovative drug for the treatment of rheumatoid arthritis. Expert Opin Biol Ther 8: 669-681, 2008.

9. Bongartz T: Tocilizumab for rheumatoid and juvenile idiopathic arthritis. Lancet 371: 961-963, 2008

10. Kudo M, Jono H, Shinriki S, Yano S, Nakamura H, Makino K, Hide T, Muta D, Ueda M, Ota K, et al: Antitumor effect of humanized anti-interleukin-6 receptor antibody (tocilizumab) on glioma cell proliferation. Laboratory investigation. J Neurosurg 111: 219-225, 2009.

11. Haura EB, Livingston S and Coppola D: Autocrine interleukin-6/interleukin-6 receptor stimulation in non-small-cell lung cancer. Clin Lung Cancer 7: 273-275, 2006.

12. Yi H, Cho HJ, Cho SM, Jo K, Park JA, Kim NH, Amidon GL, Kim JS and Shin HC: Blockade of interleukin-6 receptor suppresses the proliferation of $\mathrm{H} 460$ lung cancer stem cells. Int J Oncol 41: 310-316, 2012.

13. Koyama Y, Mitsui N, Suzuki N, Yanagisawa M, Sanuki R, Isokawa K, Shimizu N and Maeno M: Effect of compressive force on the expression of inflammatory cytokines and their receptors in osteoblastic Saos-2 cells. Arch Oral Biol 53: 488-496, 2008.

14. Jiang XP, Yang DC, Elliott RL and Head JF: Down-regulation of expression of interleukin-6 and its receptor results in growth inhibition of MCF-7 breast cancer cells. Anticancer Res 31: 2899-2906, 2011.

15. Yao X, Huang J, Zhong H, Shen N, Faggioni R, Fung M and Yao Y: Targeting interleukin-6 in inflammatory autoimmune diseases and cancers. Pharmacol Ther 141: 125-139, 2014.

16. Fizazi K, De Bono JS, Flechon A, Heidenreich A, Voog E, Davis NB, Qi M, Bandekar R, Vermeulen JT, Cornfeld M and Hudes GR: Randomised phase II study of siltuximab (CNTO 328), an anti-IL-6 monoclonal antibody, in combination with mitoxantrone/prednisone versus mitoxantrone/ prednisone alone in metastatic castration-resistant prostate cancer. Eur J Cancer 48: 85-93, 2012. 
17. Hunsucker SA, Magarotto V, Kuhn DJ, Kornblau SM, Wang M, Weber DM, Thomas SK, Shah JJ, Voorhees PM, Xie H, et al: Blockade of interleukin-6 signalling with siltuximab enhances melphalan cytotoxicity in preclinical models of multiple myeloma. Br J Haematol 152: 579-592, 2011.

18. Hsu CP, Chen YL, Huang CC, Chou CC, Liu CL, Hung CH, Kao TY and Chung YC: Anti-interleukin-6 receptor antibody inhibits the progression in human colon carcinoma cells. Eur J Clin Invest 41: 277-284, 2011.

19. Schneider MR, Hoeflich A, Fischer JR, Wolf E, Sordat B and Lahm H: Interleukin-6 stimulates clonogenic growth of primary and metastatic human colon carcinoma cells. Cancer Lett 151: 31-38, 2000.

20. Yilmaz S and Simsek I: Early intervention in the treatment of rheumatoid arthritis: focus on tocilizumab. Ther Clin Risk Manag 9: 403-408, 2013.

21. Oldfield V, Dhillon S and Plosker GL: Tocilizumab: a review of its use in the management of rheumatoid arthritis. Drugs 69: 609-632, 2009.

22. Klein B, Wijdenes J, Zhang XG, Jourdan M, Boiron JM, Brochier J, Liautard J, Merlin M, Clement C, Morel-Fournier B, et al: Murine anti-interleukin-6 monoclonal antibody therapy for a patient with plasma cell leukemia. Blood 78: 1198-1204, 1991.
23. Sato K, Tsuchiya M, Saldanha J, Koishihara Y, Ohsugi Y, Kishimoto T and Bendig MM: Reshaping a human antibody to inhibit the interleukin 6-dependent tumor cell growth. Cancer Res 53: 851-856, 1993.

24. Stärkel P, Charette N, Borbath I, Schneider-Merck T, De Saeger C, Abarca J,LeclercqI and Horsmans Y: Ras inhibition in hepatocarcinoma by S-trans-trans-farnesylthiosalicyclic acid: association of its tumor preventive effect with cell proliferation, cell cycle events and angiogenesis. Mol Carcinog 51: 816-825, 2012.

25. Sorriento D, Illario M, Finelli R and Iaccarino G: To NFאB or not to NFkB: The Dilemma on How to Inhibit a Cancer Cell Fate Regulator. Transl Med UniSa 4: 73-85, 2012.

26. Chang TP and Vancurova I: NFkB function and regulation in cutaneous T-cell lymphoma. Am J Cancer Res 3: 433-445, 2013.

27. Martin AG: NFאB anti-apoptotic or pro-apoptotic, maybe both. Cell Cycle 9: 3131-3132, 2010.

28. Viatour P,Merville MP, Bours V and Chariot A: Phosphorylation of NF-kappaB and IkappaB proteins: implications in cancer and inflammation. Trends Biochem Sci 30: 43-52, 2005. 